\title{
Growth and characterization of new oxide and fluoride crystals for optical applications
}

\author{
K SHIMAMURA*, S L BALDOCHI ${ }^{\dagger}$, NA MUJLATU, K NAKANO and T FUKUDA \\ Institute for Materials Research, Tohoku University, Sendai 980-8577, Japan \\ †Instituto de Pesquisas Energeticas e Nucleares, SP, Brazil
}

\begin{abstract}
High quality oxide and fluoride single crystals for optical applications have been grown by the Czochralski technique. Lattice parameter investigation of grown $\mathrm{Gd}_{3} \mathrm{Yb}_{x} \mathrm{Ga}_{5-x} \mathrm{O}_{12}$ suggested that this crystal will be a superior material as substrate for optical isolators with large Faraday effect. Growth conditions of $(\mathrm{La}, \mathrm{Sr})(\mathrm{Al}, \mathrm{Ta}) \mathrm{O}_{3}$ single crystals are discussed. These crystals have excellent lattice matching with GaN, a promising material for optoelectronjc devices. Ce-doped fluoride single crystals-LiCaAlF , LiYF $_{4}$ and $\mathrm{BaLiF}_{3}$-have been grown for solid state UV laser applications. Growth results and optical characterization are discussed.
\end{abstract}

Keywords. Crystal growth; Czochralski method; oxides; fluorides; UV lasers.

\section{Introduction}

The growth of single crystals has been developed over the years to meet needs for basic research and applications in several different areas. Oxide crystals are of highest importance for modern optical applications, especially as laser hosts, and as substrate or insulator materials in several devices. Fluoride single crystals, because of their unique properties such as large band gap, also present many advantages as optical materials. The crystal growth research described in this work involved the growth of high quality oxide and fluoride single crystals for optical applications. We have studied the crystal growth and properties of $\mathrm{Gd}_{3} \mathrm{Yb}_{x} \mathrm{Ga}_{5-x} \mathrm{O}_{12},(\mathrm{La}, \mathrm{Sr})(\mathrm{Al}, \mathrm{Ta}) \mathrm{O}_{3}$, and Cedoped fluorides such as $\mathrm{LiCaAlF}_{6}, \mathrm{BaLiF}_{3}$, and $\mathrm{LiYF}_{4}$ single crystals.

2. $\mathrm{Gd}_{3} \mathrm{Yb}_{x} \mathrm{Ga}_{5-x} \mathrm{O}_{12}$ (GYGG), a new substrate for optical isolators with large Faraday effect

Bi-containing iron garnet epitaxial layers grown by the LPE method, $\left(\mathrm{RE}_{1-x} \mathrm{Bi}_{x}\right)(\mathrm{Fe}, \mathrm{Ga})_{5} \mathrm{O}_{12}$ (RE: rare-earth or $\mathrm{Y}^{3+}$ ions), are attractive for magneto-optical applications such as optical isolators and recording media (Lataifeh and Lehlooh 1996). The magnitude of the Faraday rotation depends linearly on the $\mathrm{Bi}^{3+}$ concentration in the garnet epilayers. As the concentration of $\mathrm{Bi}^{3+}$ ions increases, the lattice parameter of the typical iron garnet increases linearly. Garnet single crystal substrates, with lattice parameter $\sim 12.6 \AA$, are needed in order to grow iron garnet epitaxial layers with higher $\mathrm{Bi}^{3+}$ ion concentrations. Although GGG garnet single crystals are used as sub-

*Author for correspondence strates, their small lattice parameter $(12.38 \AA)$ is a limiting factor for increase of the $\mathrm{Bi}^{3+}$ ion concentration. We have designed a new candidate, $\mathrm{Gd}_{3} \mathrm{Yb}_{x} \mathrm{Ga}_{5-x} \mathrm{O}_{12}$ (GYGG), by substituting the $A$-sites of $\mathrm{GGG}$ with $\mathrm{Yb}^{3+}$. The variation in lattice parameter in the GYGG Czochralski grown crystals and site occupation was studied.

The garnet structure can be expressed as $C_{3} A_{2} D_{3} O_{12}$, where $C, A$ and $D$ represent cations. The unit cell of the cubic system garnet structure contains 8 units of $C_{3} A_{2} D_{3} \mathrm{O}_{12}$. The $24 C, 16 \mathrm{~A}$, and $24 \mathrm{D}$ ions lie in dodecahedral ( $C$-sites), octahedral ( $A$-sites), and tetrahedral ( $D$-sites) sites, respectively. In order to enlarge the lattice parameter of the GGG single crystals, we have designed a new substance, $\mathrm{Gd}_{3} \mathrm{Yb}_{x} \mathrm{Ga}_{5-x} \mathrm{O}_{12}$ (GYGG), by substituting the $A$-sites of GGG with $\mathrm{Yb}^{3+}$.

$\mathrm{Gd}_{3} \mathrm{Yb}_{x} \mathrm{Ga}_{5-x} \mathrm{O}_{12}$ crystals were synthesized by solid state reaction, and single crystals were grown by the Czochralski technique, with several $x$ values. Figure 1 shows the variation of the lattice parameter, $a_{\mathrm{sS}}$, (solid state reaction) and $a_{\mathrm{CZ}}$ (CZ single crystals) as a function of the $x$ value. The lattice parameter $a$ increased with the concentration of $\mathrm{Yb}^{3+}$, corresponding to $x$, the variation being almost linear up to $x=1$.

Figure 2 shows GYGG $(x=2)$ single crystals pulled at the rate of $0.6 \mathrm{~mm} / \mathrm{h}$ with a rotation rate of $10 \mathrm{rpm}$. Lattice parameters measured for single crystals plotted in figure 1 , ranged from $12.38 \AA$ to $12.68 \AA$, i.e. in the vicinity of the desired lattice constant of $12 \cdot 62 \AA$. GYGG single crystals had a lattice parameter over $12.6 \AA$, when $x$ was larger than 1 .

Chemical composition and structural analysis of the GYGG $(x=2)$ single crystal showed that the $C$-site was occupied by $\mathrm{Gd}^{3+}$ and $\mathrm{Yb}^{3+}$, the $A$-site by $\mathrm{Yb}^{3+}$ and $\mathrm{Ga}^{3+}$ ions, and the $D$-site only by $\mathrm{Ga}^{3+}$ ions. Based on the 
result of the structural analysis, the formula of the GYGG garnet composition can be expressed as $\{\mathrm{Gd}, \mathrm{Yb}\}_{3}[\mathrm{Yb}, \mathrm{Ga}]_{2}(\mathrm{Ga})_{3} \mathrm{O}_{12}$, where curly brackets, square brackets, and parentheses enclose the $C$-, $A$-, and $D$-sites, respectively.

The ionic radius of $\mathrm{Yb}^{3+}$ is smaller than that of $\mathrm{Gd}^{3+}$, and larger than that of $\mathrm{Ga}^{3+}$. In GYGG single crystals grown by the $\mathrm{CZ}$ method, $\mathrm{Yb}^{3+}$ preferentially substitutes $\mathrm{Gd}^{3+}$, which occupies $C$-sites in the $x$ range from 0 to 0.5 . For larger $x, \mathrm{Yb}^{3+}$ starts to substitute $\mathrm{Ga}^{3+}$ in $A$-sites selectively, and then substitutes $\mathrm{Gd}^{3+}$ in $C$-sites again when $x$ exceeds $\sim 1 \cdot 7$. That is, each site of the $\mathrm{CZ}$ grown GYGG single crystals is occupied by cations in a different manner depending on the $x$ range. The variation of $a_{\mathrm{SS}}$ has a similar tendency.

\section{3. (La,Sr)(Al,Ta) $\mathrm{O}_{3}$ (LSAT), a new substrate for GaN epitaxial growth}

Due to its wide direct bandgap and high thermal stability, $\mathrm{GaN}$ is a promising material for optoelectronic devices such as diodes and lasers emitting from blue to ultraviolet

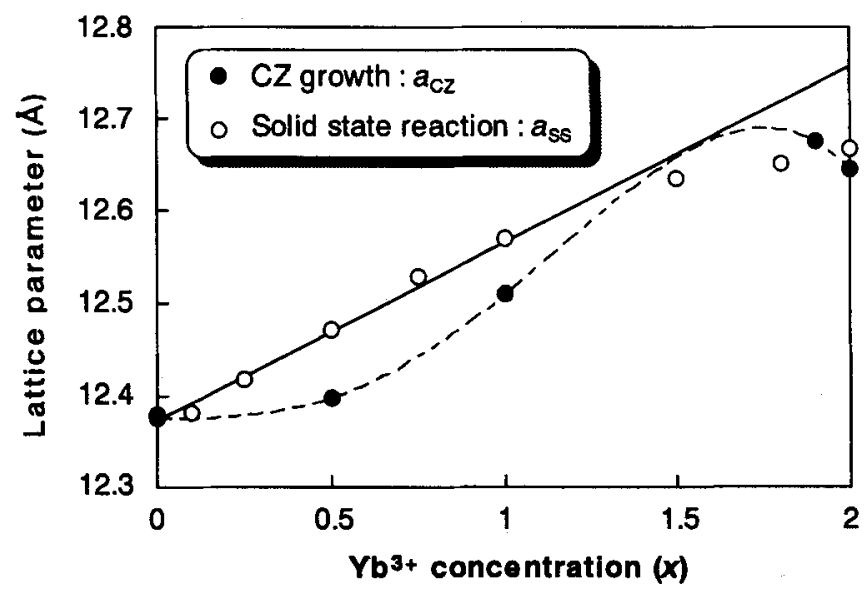

Figure 1. Dependence of the lattice parameter on the $\mathrm{Yb}^{3+}$ concentration, $x$, in GYGG crystals.

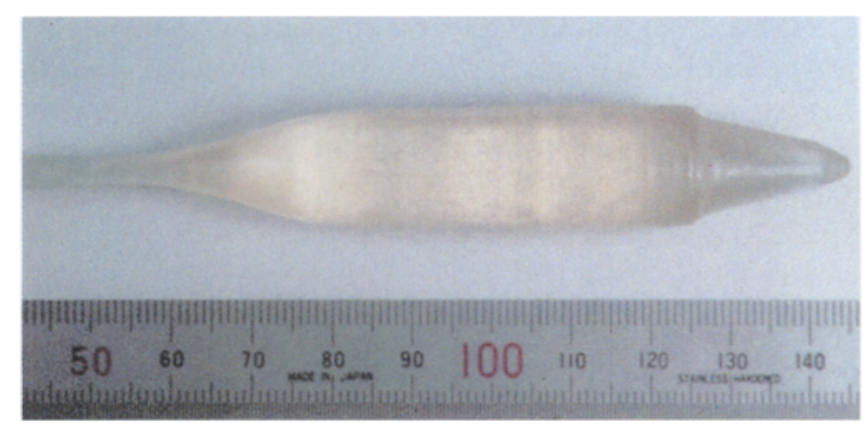

Figure 2. As-grown GYGG single crystals pulled at rates of $1.0 \mathrm{~mm} / \mathrm{h}$ and $0.6 \mathrm{~mm} / \mathrm{h}$. wavelength regions, as well as for electronic devices operating at high temperatures. It has been reported that $\mathrm{GaN}$ layers could be grown on sapphire substrates by MOCVD, (Morkoc et al 1994; Nakamura et al 1994, 1995, 1996; Akasaki et al 1995). However, dislocation densities in these layers are very large, because of the large lattice mismatch between the epitaxial layers and the substrates (14-16\%) (Lester et al 1995; Osinski and Barton 1996). These dislocations have recently been shown to strongly affect the lifetime of continuous wave blue laser diodes (Amano et al 1986). We have found a new material: $(\mathrm{La}, \mathrm{Sr})(\mathrm{Al}, \mathrm{Ta}) \mathrm{O}_{3}$ (LSAT) as a substrate for GaN epitaxial growth. LSAT single crystals have been grown by the Czochralski technique, and lattice parameter and crystal quality were investigated.

Figure 3 shows an as-grown LSAT single crystal pulled along the $\langle 111\rangle$ direction at a pulling rate of $1 \mathrm{~mm} / \mathrm{h}$. Figure $4 \mathrm{a}$ shows a cross-polarized light image of the wafer cut parallel to the growth direction at the shoulder part of the crystal shown in figure 3. The growth interface was fairly convex towards the melt. The existence of a core in the central region was clearly shown by the bright area in the image. Figure $4 \mathrm{~b}$ shows a similar image of the wafer prepared from the crystal grown under the same growth conditions, except that the rotation rate was $40 \mathrm{rpm}$ rather than $10 \mathrm{rpm}$. Although the growth interface at $40 \mathrm{rpm}$ was still convex towards the melt, the core observed in figure $4 \mathrm{a}$ disappeared, and the shape of the growth interface became smooth. This suggests that a higher crystal rotation speed is preferable in order to decrease the stress in the grown crystals.

The lattice parameter of the LSAT single crystal was $7.735 \AA$. The lattice parameter was almost constant along the growth axis. The (111) plane of the LSAT crystals is a close-packed plane of the anion framework, which forms the primitive cubic lattice, and thus the $\langle 111\rangle$ direction is the body-diagonal direction of this cubic cell. The surface structure, therefore, has hexagonal symmetry. The lattice parameter $a_{\text {hex }}$ of the LSAT (111) plane, considered as a 2-dimensional hexagonal crystal, is given by

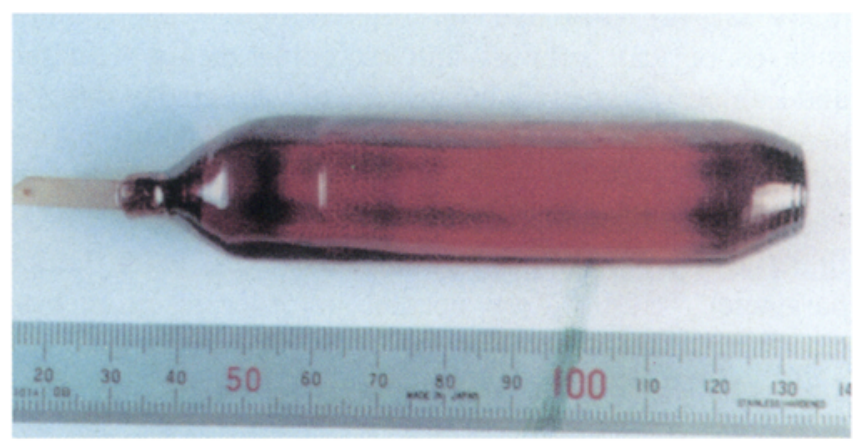

Figure 3. As-grown LSAT single crystal pulled along the 〈111) orientation. 


$$
a_{\text {hex }}=\sqrt{6} / 3 \cdot a_{\text {cub }}
$$

where $a_{\text {cub }}$ is the conventional lattice parameter of the cubic crystal. Since the measured $a_{\text {cub }}$ of LSAT was $7.735 \AA, a_{\text {hex }}$ was calculated to be $6.316 \AA$, which was close to twice the corresponding lattice parameter of GaN (3.160-3.190 $)$. The lattice mismatch was calculated to be $0.06-1.00 \%$.

\section{Ce-doped fluorides for UV laser applications}

Coherent optical sources in the ultraviolet wavelength region are useful for many practical applications such as medicine, semiconductor processing, optical communications and remote sensing (Sarukura et al 1995). Different kinds of dye lasers and tunable colour centre lasers have been investigated as UV sources, but their applications are still restricted because they are limited concerning temperature operation and crystal deterioration. Recently, Ce-doped $\mathrm{LiCaAlF}_{6}(\mathrm{Ce}: \mathrm{LiCAF})$ crystals
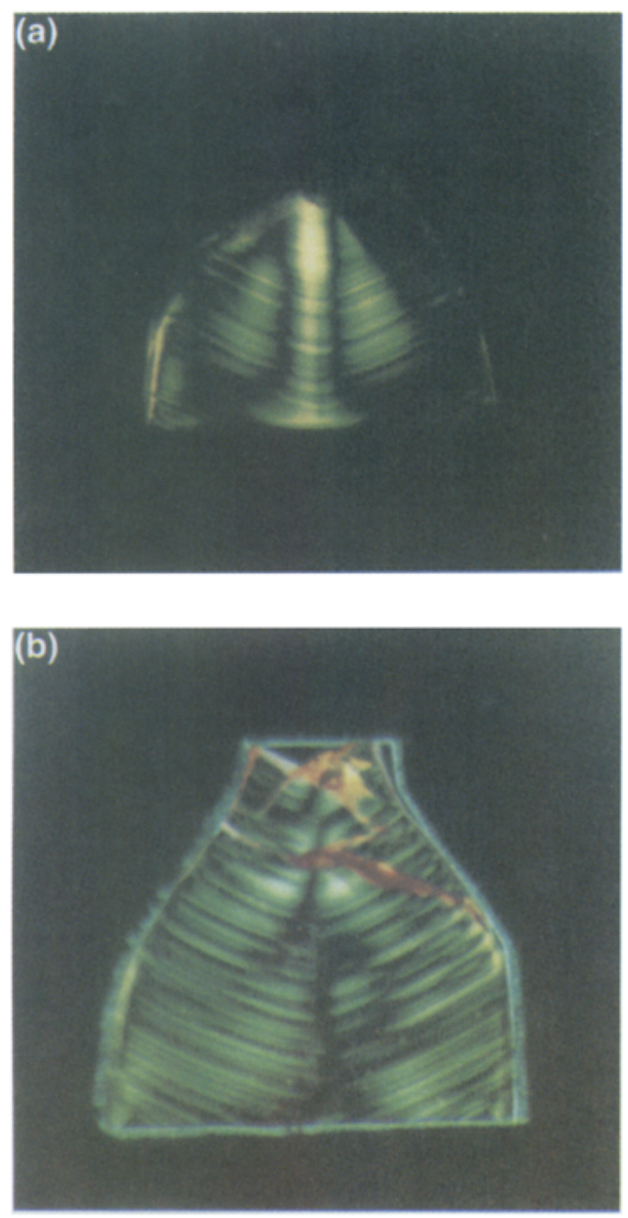

Figure 4. Cross-polarized light image of waters from the shoulder part of the crystal in figure 3 , cut parallel to the growth direction. The crystal rotation rate was $10 \mathrm{rpm}$ (a) and $40 \mathrm{rpm}$ (b). have been reported as candidates for UV solid state lasers (Dubinskii et al 1993; Marshall et al 1994). This matrix can be directly pumped by the fourth harmonic of a Nd : YAG laser and is suitable for tunable all solid state lasers in UV wavelength region. Very few UV laser materials appropriate for direct pumping are known. The search for new materials includes studies on $\mathrm{BaLiF}_{3}$ (BLF) (Dubinski et al 1997), a new candidate material, and known laser host as $\mathrm{LiYF}_{3}$ (YLF). We describe the growth of LiCAF: $\mathrm{Ce}, \mathrm{YLF}: \mathrm{Ce}$ and BLF:Ce single crystals by the Czochralski technique. Laser performance and tunability were demonstrated for LiCAF:Ce.

We have studied the LiCAF : Ce growth, under inert and fluorinating atmospheres, investigated the deviation of composition and $\mathrm{Ce}^{3+}$ incorporation. Commercially available $\mathrm{AlF}_{3}, \mathrm{CaF}_{2}$ and $\mathrm{LiF}$ powders, of high purity $(>99.99 \%)$, were used as the starting materials, $\mathrm{CeF}_{3}$ powder (>99.99\%), was used to dope the crystal in the range 1-2 mol\%. A glassy carbon crucible was used for $\mathrm{CZ}$ growth. All crystals were grown from $a$-axis oriented seed crystals. The pulling rate was $1 \mathrm{~mm} / \mathrm{h}$ and the rotation rate was $10 \mathrm{rpm}$. The $\mathrm{Ce}^{3+}$ doping level in the grown crystals was measured, and the distribution coefficient of $\mathrm{Ce}^{3+}\left(k_{\mathrm{Ce}}\right)$ in LiCAF was estimated as 0.01 .

Figure 5 shows an $\mathrm{Ce}: \mathrm{LiCAF}$ single crystal growth from a stoichiometric charge under inert atmosphere. The crystal surface is covered by white foreign substances. Figure 6 shows a wafer, which was cut perpendicular to the growth axis and polished. It is interesting to note that the inside part is transparent and without cracks or macroscopic inclusions.

Figure 7a shows a SEM image taken of the foreign substance stuck to the crystal surface. It is formed mainly of volatile fluorides such as $\mathrm{LiAlF}_{4}$, and small amounts of $\mathrm{AlF}_{3}$ and $\mathrm{LiF} . \mathrm{CaF}_{2}$ were also detected in these foreign substances. The presence of oxides and oxyfluorides such as $\mathrm{Al}_{2} \mathrm{O}_{3}, \mathrm{Al}_{4} \mathrm{LiO}_{6} \mathrm{~F}$ and $\mathrm{CeOF}$ was also detected. Figure $7 \mathrm{~b}$ shows a SEM image of the spherical inclusions appeared just close to the surface. These spherical inclusions were

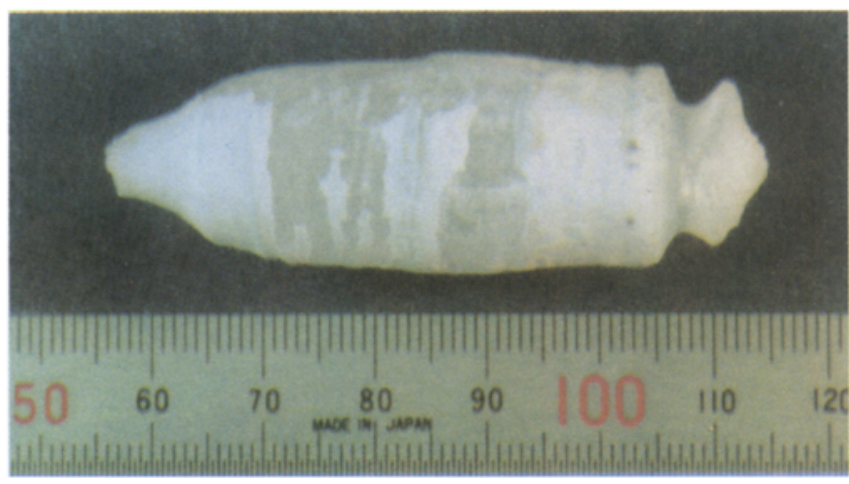

Figure 5. As-grown LCAF single crystal pulled along the a-axis. 
composed of the same compounds as the above foreign substance.

We can conclude that the formation of these inclusions resulted from evaporation of the melt components and oxide or oxyfluoride formation in the melt because of the presence of water and oxygen in the system or in the raw materials. $\mathrm{AlF}_{3}$ and $\mathrm{LiF}$ have high vapourization pressures; the evaporation of these components shifts the melt composition in the $\mathrm{CaF}_{2}$-rich direction. The excess $\mathrm{CaF}_{2}$ in the melt aggregates and sticks to the growing crystal surface, and/or reacts with other contaminants present in the melt, such as oxides and oxifluorides produced from water and/or oxygen not completely eliminated from the growth system or from the raw materials.

Samples of the central part of the grown crystals were used in preliminary tests of laser action. A schematic diagramme of the $\mathrm{Ce}: \mathrm{LiCAF}$ laser resonator is shown in figure 8 . The Ce: LiCAF crystal is located between the two cavity mirrors. There is no coating on the parallel end faces of the crystal that are perpendicular to the optical axis of the resonator. The fourth harmonic of a Q-switched Nd : YAG laser is used as the pumping source. Figure 9 shows the obtained output energies at $289 \mathrm{~nm}$ as a function of the absorbed pump energy at $266 \mathrm{~nm}$. High-energy pulse generation from a Ce : LiCAF laser operating at $289 \mathrm{~nm}$ wavelength was obtained, at a repetition rate of $10 \mathrm{~Hz}$, with up to $30.5 \mathrm{~mJ}$ output energy and slope efficiency $39 \%$. This laser performance is the highest ever reported for $\mathrm{Ce}: \mathrm{LiCAF}$ crystals, to our knowledge.

Figure 10 shows a LiCAF : $\mathrm{Ce}: \mathrm{Na}$ crystal grown under $\mathrm{CF}_{4}$ atmosphere from a nonstoichiometric composition. $\mathrm{An} \mathrm{AlF}_{3}$ and $\mathrm{LiF}$-rich composition was used to compensate the evaporation loss. It is well known, from the works of Pastor and Arita (1975), that $\mathrm{CF}_{4}$ reacts with water

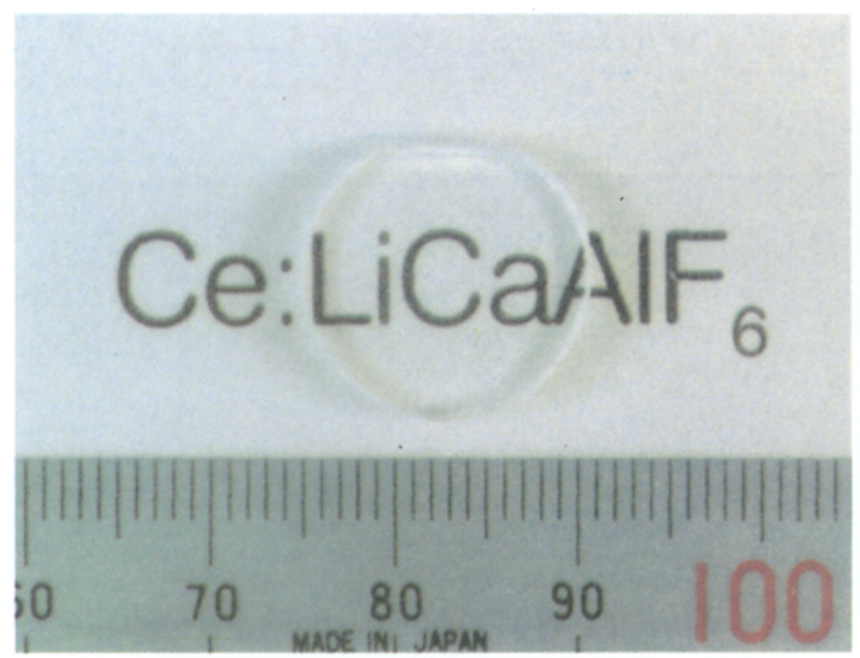

Figure 6. As-grown wafer cut perpendicular to the growth axis. in the environment, resulting in $\mathrm{HF}$ and $\mathrm{CO}_{2}$, yielding a double effect of purification by elimination of waterrelated impurities and generating a slightly fluorinated atmosphere which also acts on oxygen-derived impurities. New laser measurements with samples prepared from the crystal shown in figure 10 are now under investigation.

The discovery of the LiCAF: Ce properties for direct UV pumping stimulated several research groups to investigate the potential of other fluoride hosts as laser media in the UV region. BLF : Ce and YLF : Ce crystals are indicated as promising candidates for a tunable UV laser. BLF is an inverse perovskite material with cubic structure (space group $O_{\mathrm{h}}^{\mathrm{t}}-P m 3 m$ ) where the monovalent ion $\mathrm{Li}^{+}$is at the centre of a $\mathrm{F}_{6}$ octahedron and the $\mathrm{Ba}^{2+}$ divalent ions are in the 12 -fold environment site, resulting in a different field interaction from classic perovskite structure. YLF crystals are well known laser hosts, usually doped with rare earth ions as $\mathrm{Nd}^{3+}, \mathrm{Er}^{3+}, \mathrm{Ho}^{3+}$
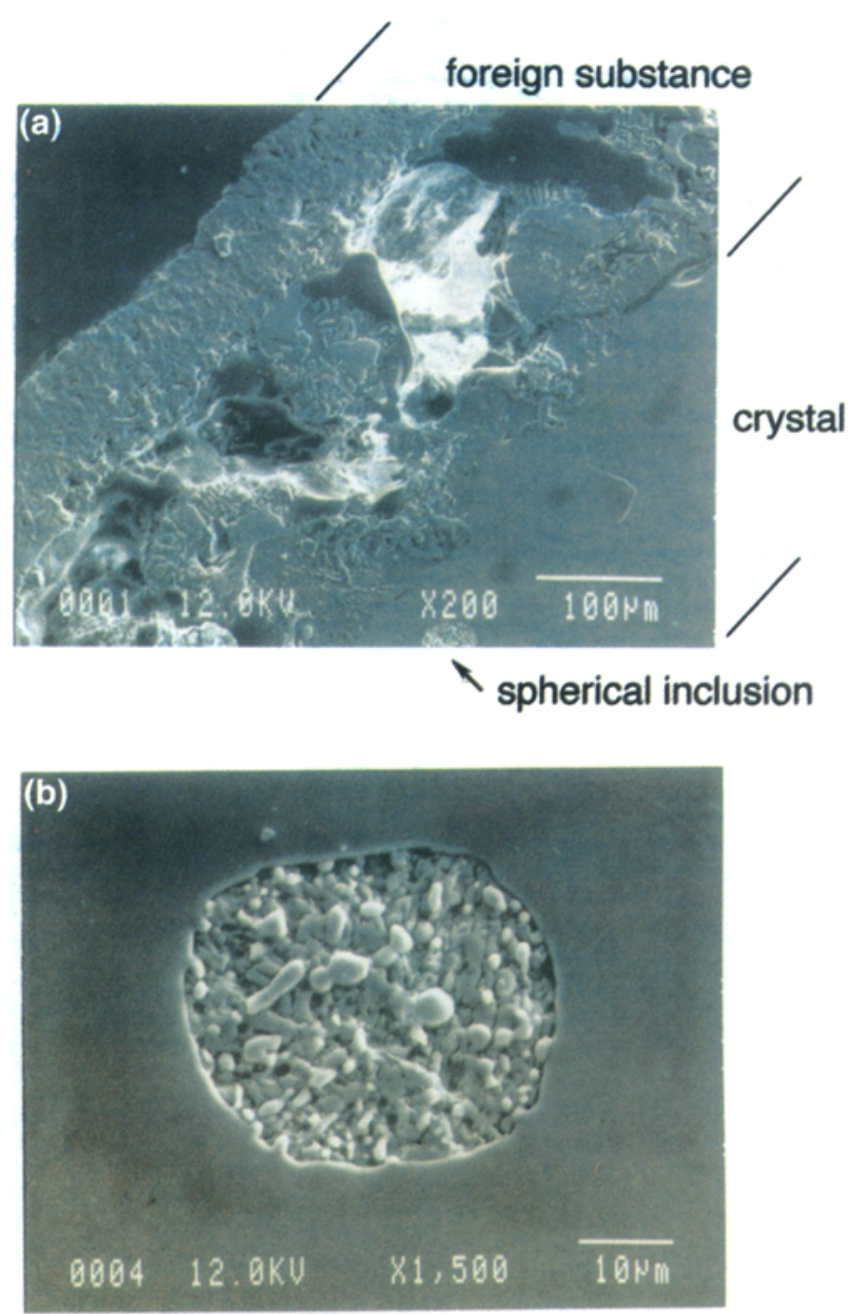

Figure 7. SEM images of (a) a cross-section of the grown crystal with foreign substances on the crystal surface, and (b) a spherical inclusion observed close to the grown crystal surface. 
or $\mathrm{Tm}^{3+}$. It is a scheelite material with tetragonal structure (space group $C_{4 \mathrm{~h}}^{6} 0 I_{1 / \mathrm{a}}^{4}$ ).

Both BLF and YLF melt incongruently, and a single crystal must be grown from a nonstoichiometric melt to avoid precipitation of other phases. We have grown large and optically high-quality crystals of Ce-doped BLF and Ce-doped YLF by Czochralski pulling under $\mathrm{CF}_{4}$ reactive atmosphere (figure 11). Commercially available $\mathrm{YF}_{3}$, $\mathrm{BaF}_{2}$ and LiF powders, of high purity $(>99.99 \%)$, were used as the starting material. $\mathrm{CeF}_{3}$ powder (>99.99\%), was used to dope the crystal in the range 1-2 mol\%. A platinum crucible was used for $\mathrm{CZ}$ growth. The pulling rate was $1 \mathrm{~mm} / \mathrm{h}$ and the rotation rate was $15 \mathrm{rpm}$.

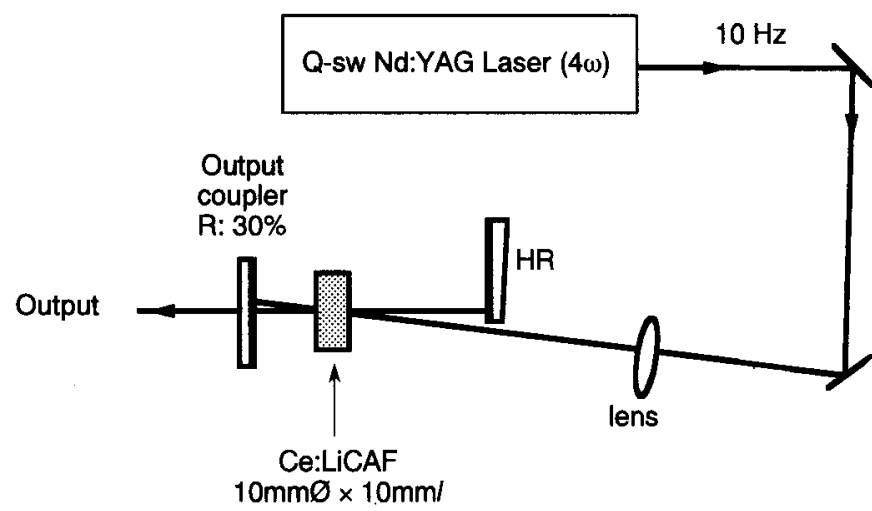

Figure 8. Experimental setup of a high-energy Ce:LiCAF laser.

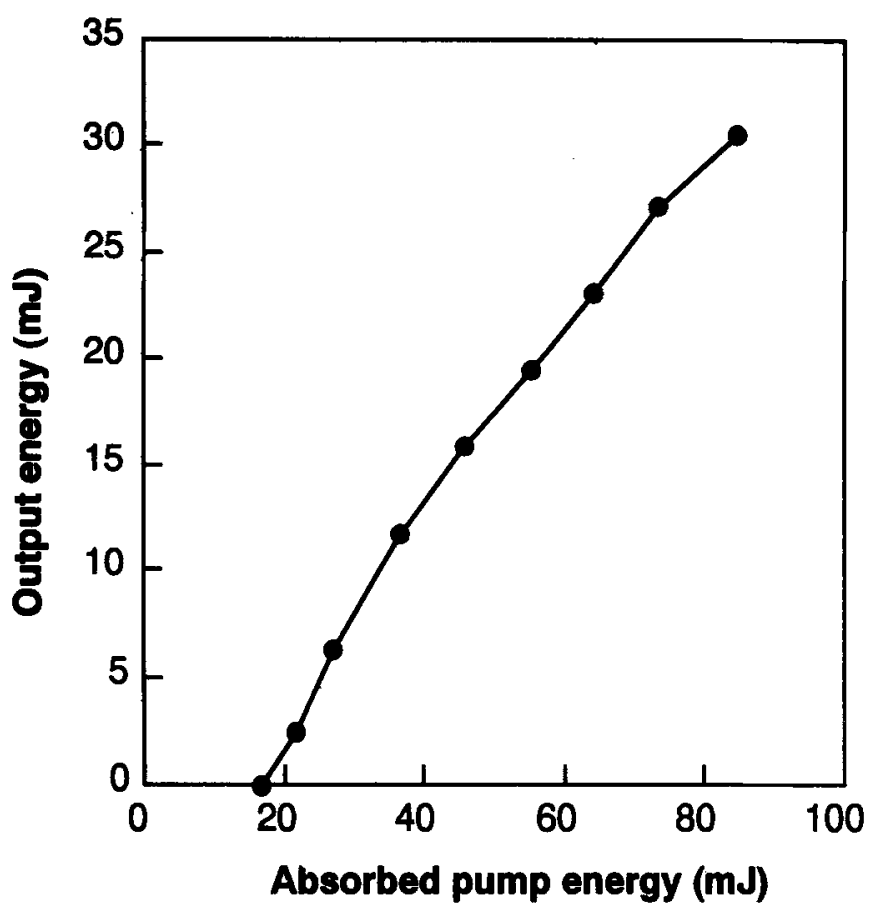

Figure 9. Laser output energy as a function of absorbed pump energy in a $\mathrm{Ce}: \mathrm{LiCaAIF}_{6}$ sample.
Figure 12 shows an IR transmission spectrum of both crystals. In fluoride crystals, free $\mathrm{OH}^{-}$radicals present absorption bands in the region $4000-3500 \mathrm{~cm}^{-1}$; from the figure we can confirm that for the BLF : Ce crystal the $\mathrm{CF}_{4}$ atmosphere eliminates all traces of $\mathrm{OH}^{-}$

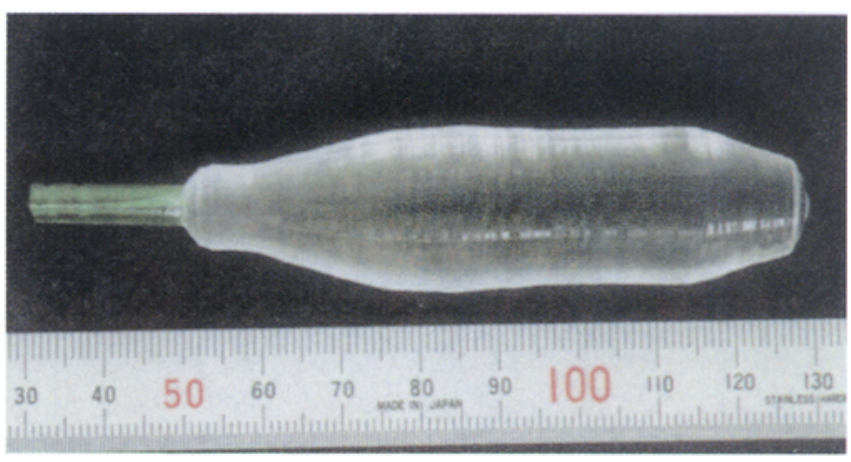

Figure 10. As-grown LiCAF: $\mathrm{Ce}: \mathrm{Na}$ crystal grown under $\mathrm{CF}_{4}$ atmosphere.

\section{K}

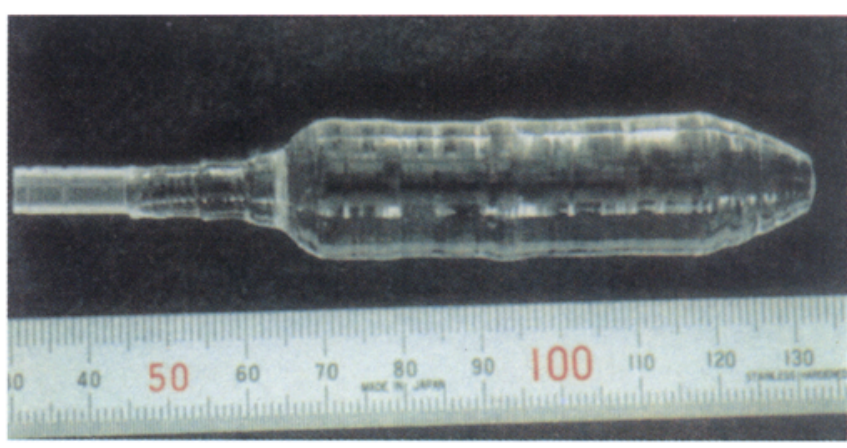

Figure 11. As-grown $\mathrm{YLF}: \mathrm{Ce}$ crystal grown under $\mathrm{CF}_{4}$ :tmosphere.

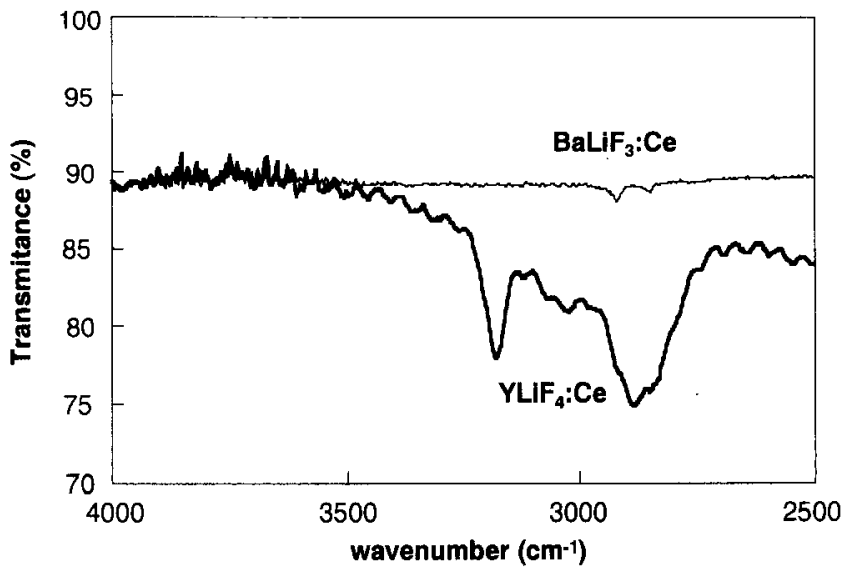

Figure 12. IR transmission spectra of $10 \mathrm{~mm}$ polished samples of BLF : Ce and YLF : Ce. 


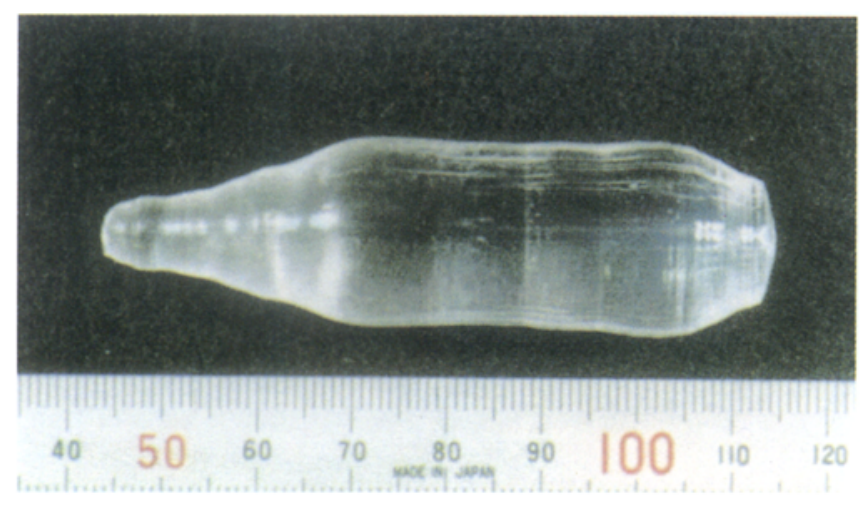

Figure 13. As-grown BLF : $\mathrm{Ce}: \mathrm{Na}$ crystal grown under $\mathrm{CF}_{4}$ atmosphere.

impurities. We observed only small bands in the range $3000-2800 \mathrm{~cm}^{-1}$ that can be attributed to $\mathrm{HCO}^{-}$molecule. In the case of YLF:Ce, there is no trace of $\mathrm{OH}^{-}$ substitutional defects, but we detected the presence of complexes in the range $3200-2800 \mathrm{~cm}^{-1}$. These bands were also observed in YLF : $\mathrm{Er}: \mathrm{OH}^{-}$co-doped crystals (Ranieri et al 1996). The $\mathrm{OH}^{-}$molecules dissociate during the growth of the YLF crystal, producing $\mathrm{O}_{m} \mathrm{H}_{n}$ complexes that absorb in a wide spectral range from 4000 to $2400 \mathrm{~cm}^{-1}$. We also noted in this crystal the presence of $\mathrm{HCO}^{-}$. The presence of this molecule is attributed to carbon contamination from the commercial fluorides, but also can result from $\mathrm{CF}_{4}$ reaction products. The presence of $\mathrm{Me}(\mathrm{OH})_{2}$ complexes, characterized by narrow bands in the range $3600-3550 \mathrm{~cm}^{-1}$, and usually detected on commercially RE-doped YLF, was not observed.

Usually, oxygen-related centres result in scattering, but we have not detected scattering in our samples. It is important to note that the IR spectrum of figure 12 was measured in large samples $(10 \mathrm{~mm})$ with high resolution. The concentrations of these impurities are very low. The dependence of YLF : Ce and BLF : Ce crystal optical quality on the concentration of these complexes is not yet established, but it is supposed that their presence should not affect UV laser performance.

The measured $\mathrm{Ce}^{3+}$ concentration in the BLF : Ce crystal was equal to 0.011 at\%. The low incorporation of $\mathrm{Ce}^{3+}$ ions in the $\mathrm{BaLiF}_{3}$ host is probably influenced by the different in valence state between these ions and available substitutional sites in the crystal. Theoretically, cerium ions can be introduced in the crystal in more than one valence state with formation of compensating defects, such as vacancies, in the host lattice. The simplest measure to stabilize the valence state, and increase the $\mathrm{Ce}^{3+}$ concentration, is to co-dope the crystal with a second impurity or a charge-compensating additive. Figure 13 shows a $\mathrm{BaLiF}_{3}: \mathrm{Ce}: \mathrm{Na}$ grown under $\mathrm{CF}_{4}$ atmosphere. The boule is transparent, without inclusions on the surface or inside. The measurement of the $\mathrm{Ce}^{3+}$ concentration in co-doped crystals showed a lower $\mathrm{Ce}$ incorporation when compared to the singly doped one. The addition of $\mathrm{Na}^{+}$ions presented an inverse result from the expected one: sodium decreased the cerium incorporation in the BLF host.

\section{Summary}

Various new oxide and fluoride crystals were developed. Detailed study of the Czochralski crystal growth process and characterization of the grown crystals allowed the design of new materials for optical applications, such as GYGG, a good candidate as substrate for the growth of iron garnet epitaxial layers with high $\mathrm{Bi}^{3+}$ ion concentrations, and LSAT, having high potential as a substrate for the growth of high-quality GaN epitaxial layers with low dislocation density. Study of purification and crystal growth process resulted in the growth of large crystals of LiCAF : $\mathrm{Ce}: \mathrm{Na}, \mathrm{BLF}: \mathrm{Ce}$ and YLF : Ce with good optical quality. High-energy pulse generation $(30.5 \mathrm{~mJ})$ was obtained from a Ce: LiCAF laser. This laser performance proves that $\mathrm{Ce}: \mathrm{LiCAF}$ is a promising material for high-energy UV pulse generation combined with a high-power, Q-switched Nd : YAG laser.

\section{Acknowledgements}

The authors would like to express sincere thanks to $\mathrm{Dr}$ $S$ Durbin, Institute for Materials Research, Tohoku University, for his critical reading of the manuscript and fruitful discussions. The authors are also indebted to $\mathrm{Mr}$ Y Murakami, Institute for Materials Research, Tohoku University, and Dr N Sarukura, Institute of Molecular Science, for their help in carrying out the chemical composition analysis and laser experiments.

\section{References}

Akasaki I, Amano H, Sato S, Sakai H, Tanaka T and Koike M 1995 Jpn J. Appl. Phys. 34 L1517

Amano H, Sawaki N, Akazaki I and Toyoda Y 1986 Appl. Phys. Lett. 48353

Dubinskii M A, Semashko V V, Naumov A K, Abdulsabirov R Y and Korableva S L 1993 Laser Phys. 3216

Dubinski M A, Schepler K L, Semashko V V, Abdulsabirov R Y, Galjautdinov B M, Korableva S L and Naumov A K 1997 OSA TOPS, Advanced Solid State Lasers Vol. 10, p. 30-34

Lataifeh M S and Lehlooh A F D 1996 Solid State Commun. 97805

Lester S D, Ponce F A, Craford M G and Steigerwald D A 1995 Appl. Phys. Lett. 661249

Marshall C D, Payne S A, Speth J A, Krupke W F, Quarles G J, Castillo V and Chai B H T 1994 J. Opt. Soc. Am. B11 2054

Morkoc H, Strite S, Gao G B, Lin M E, Sverdlov B and Bums M 1994 J. Appl. Phys. 761363 
Nakamura S, Mukai T and Senoh M 1994 Appl. Phys. Lett. 64 1687

Nakamura S, Senoh M, Iwasa N and Nagahama S 1995 Jpn J. Appl. Phys. 34 L797

Nakamura S, Senoh M, Nagahama S, Iwasa N, Yamada T, Matsushita T, Kiyoku H and Sugimoto Y 1996 Jpn J. Appl. Phys. 35 L74
Osinski M and Barton D L 1996 Proceedings of the international symposium on blue laser and light emitting diodes p. 217

Pastor A C and Arita K 1975 Mater. Res. Bull. 10493

Ranieri I M et al 1996 J. Crystal Growth 166423

Sarukura N et al 1995 IEEE J. Selected Topics in Quantum Electronics 1792 